\title{
An Analysis of Industrial Linking Technologies and the Development Direction of the Green Chemical Industry
}

\author{
Li Jin, Hu Shanying, Chen Dingjiang, Song Xiaoxu, Zhang Qun, Fan Jiongming, Li Guangyao, Ma Shujie, Jin Yong
}

Center for Industrial Ecology, Department of Chemical Engineering, Tsinghua University, Beijing 100084, China

\begin{abstract}
Over the course of the 13th Five-Year Plan, significant progress has been made in the green development of China's chemical industry, not only by increasing product yields, but also by significantly reducing resource consumption, energy consumption, and pollution emission. Green manufacturing is necessary for achieving sustainable development in China. In order to realize green development of the chemical industry, it is necessary to pay attention to not only green manufacturing in the chemical industry, but also to green coordinated development when the chemical industry partners with other industries and society. Based on the current situation and development directions of the green chemical industry in China, the authors put forward key technologies in the relationship between the chemical industry and the green manufacturing industry, and demonstrate a typical pattern analysis using five business cases.
\end{abstract}

Keywords: green manufacturing; industrial linking technology; strategy; chemical industry

\section{Introduction}

Nowadays, with the multiple constraints of resources, energy, and environment, the circular and cascade utilization of resources and energy has become an urgent requirement in the development of the chemical industry. In May 2015, the Chinese government issued the Made in China 2025 plan, which pointed out that the green transformation of the traditional manufacturing industries, such as the chemical industry, should be widely promoted. Green manufacturing requires the environmental impact and natural resource utilization rate to be given primary consideration in the manufacturing process, so as to ensure that the resource utilization rate for the whole lifecycle reaches its highest point, and any adverse impact on the environment is reduced to the lowest possible amount [1].

Since the concepts of "green manufacturing" and "green chemicals" were proposed, the development of a green chemical industry in China has achieved initial success in related fields. In 1995, China put forward the subject of "green chemistry and technology," and actively promoted the related research of the green chemical industry and its development [2,3]. Currently, the research into the relevant technology includes the whole lifecycle of "resources-process-product-use-integration" [4], including aspects of chemical production innovation, technology innovation, zero emission of chemical production, and so on $[5,6]$. In addition, the development of information technology and intelligent technology has also effectively promoted the development of the green chemical industry to the traditional chemical industry in the areas of green products and hazard warning [7].

The development of the green chemical industry is faster in developed countries. The US state of California requires green chemical activity to promote the development of the green chemical industry. Britain has created a green chemical network

Received date: June 20, 2017; Revised date: July 10, 2017

Corresponding author: Hu Shanying, Center for Industrial Ecology, Department of Chemical Engineering, Tsinghua University, Professor. Major research fields include design, analysis and integration methods of eco-industrial system and theory and practice of circular economy. E-mail: hxr-dce@tsinghua.edu.cn.

Funding program: CAE Advisory Project “Research on Manufacturing Power Strategy (Phase II)" (2015-ZD-15)

Chinese version: Strategic Study of CAE 2017, 19 (3): 072-079

Cited item: Li Jin et al. An Analysis of Industrial Linking Technologies and the Development Direction of the Green Chemical Industry. Strategic Study of CAE, https:// doi.org/10.15302/J-SSCAE-2017.03.011 
that combines colleges, factories, and retailers to promote the development of the green chemical industry. Japan has invested a lot in the establishment of a new energy efficiency facility and upgrading existing facilities, as well as other energy saving and emission reducing actions. The European Union enacted the REACH regulation on the management and control of the negative effects of all chemical substances throughout their entire lifecycle. Compared with the developed countries in the world, the development and application of the green chemical industry in China is still far from adequate.

Because chemical products are widely used in various fields, such as industry, agriculture, people's daily lives, and so on, the chemical industry and various other industries can achieve certain degrees of industrial links. The green manufacturing of the chemical industry involves not only the green manufacturing of the chemical industry itself, but also the green coordinated development of the chemical industry and other industries. Therefore, its industrial links include three aspects: the construction of an intra-industry chain, the coordinated development of green manufacturing between industries, and the partnership between industry and society. Realizing the industrial link to green manufacturing in the chemical industry is of great significance, and can accelerate the new round of technological upgrading and mode adjustment in China's chemical industry.

The general outline of this article is shown in Fig. 1.

\section{The present situation of green manufacturing in China's chemical industry}

With the rapid development of chemical technology in China, the production of chemical products is now at the forefront of the world. Since the beginning of the twenty-first century, China's chemical industry has maintained a trend of good growth. The total output value of the chemical industry as a proportion of the overall total industrial output value has remained around $10 \%$. In 2015 , the output value of the chemical industry accounted for $11.9 \%$ of the total industrial output of the country, placing it first out of 36 industrial sectors in the country. In the last ten years, this has increased at an annual rate of $18 \%$, making an important contribution to the development of the country's economy. With the development and popularization of cleaner production, energy saving, emission reduction, and chemical reaction process intensification technology in recent years, the green development of the chemical industry in China has achieved initial success.

In recent years, the chemical industry has made remarkable progress in the production of major products, energy saving, consumption reduction, emission reduction, and so on.

(1) Production of major products. The production of methanol in 2015 was $3.93 \times 10^{7}$ t, up $149.7 \%$ from 2010 . Ethylene production was $1.714 \times 10^{7} \mathrm{t}$, up $20.8 \%$ from 2010 . The production of soda increased to $2.592 \times 10^{7}$ t, up $27.8 \%$ from 2010 . The production of caustic soda was $3.028 \times 10^{7}$ t, up $45.1 \%$ from 2010 . The output of phosphate rock was $1.42 \times 10^{8}$ t, up $108.9 \%$ from 2010. The output of N, P, K and K fertilizer was $7.432 \times 10^{7}$ tons, up $17.3 \%$ from 2010 (Table 1) [8].

(2) Resource consumption. The intensity of phosphate rock consumption during the 12th five-year cycle showed a steady trend, from $1.28 \times 10^{-5} \mathrm{t}$ /yuan in 2011 to $1.29 \times 10^{-5} \mathrm{t} /$ yuan in 2015. The consumption of pyrite fell from $1.6 \times 10^{-6} \mathrm{t} / \mathrm{yuan}$ in 2011 to $1.23 \times 10^{-6} \mathrm{t} /$ yuan in 2015 , a decline of $23.13 \%$ (Table 2).

(3) Energy consumption. By 2014, the total amount of energy consumption in the chemical industry reached $5.28 \times 10^{8}$ tonnes

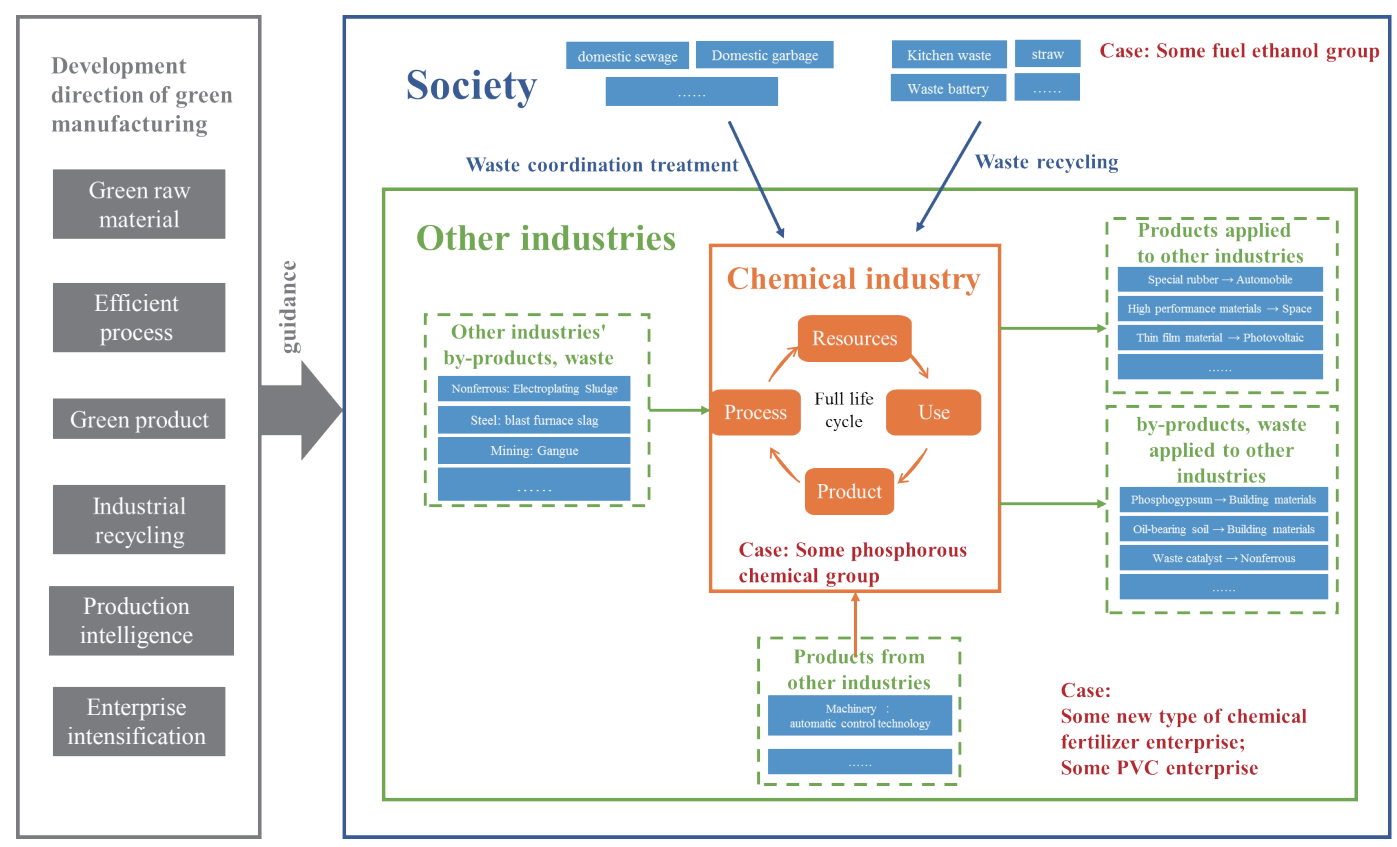

Fig. 1. The framework of green manufacturing development for chemical industry. 
of coal equivalent (tce), which increased by about $1.64 \times 10^{8}$ tce compared to 2011 . The intensity of energy consumption decreased from $6 \times 10^{-5} \mathrm{t} / \mathrm{yuan}$ in 2011 to $5.6 \times 10^{-5} \mathrm{t}$ /yuan in 2014 , and the decrease reached $6.67 \%$. The consumption of ethylene energy decreased from the $880.7 \mathrm{~kg}$ standard coal $/ \mathrm{t}$ in 2010 to the $816.3 \mathrm{~kg}$ standard coal $/ \mathrm{t}$ in 2014 , a decline of $7.31 \%$. The energy consumption of calcium carbide dropped from the $1100 \mathrm{~kg}$ standard coal / $\mathrm{t}$ in 2010 to the 1010.2 standard coal / $\mathrm{t}$ in 2014, a decrease of $8.2 \%$. The energy consumption of synthetic material decreased from the $471.5 \mathrm{~kg}$ standard coal / $\mathrm{t}$ in 2010 to the $/ \mathrm{t}$ of $367.7 \mathrm{~kg}$ in 2014, a decrease of 22.6\% (Table 3).

(4) Pollutant discharge. The total emissions of solid waste in China's chemical industry decreased rapidly during the 12th five-year period, with emissions of pollutants declining from $1.396 \times 10^{5} \mathrm{t}$ in 2011 to $6.07 \times 10^{4} \mathrm{t}$ in 2015 , a rate of decline of $56.5 \%$ (Table 4 ). With the development of recycling technology for chemical plant wastewater and the improvement in the recovery and utilization rate of wastewater in recent years, the emission intensity of wastewater is obviously decreasing. The discharge intensity of waste water dropped from $5.20 \times 10^{9} \mathrm{t}$ in 2010 to $3.46 \times 10^{9} \mathrm{t}$ in 2015 , a decrease of $33.5 \%$. With the improvement and development of COD emission reduction and control technology, the emission intensity of COD continued obvious decline between 2010 and 2015. The total emission of COD declined from $3.28 \times 10^{5} \mathrm{t}$ in 2010 to $2.43 \times 10^{5} \mathrm{t}$ in 2015 , a decrease of $17.7 \%$. The total emission of nitrogen compounds decreased from $9.27 \times 10^{4} \mathrm{t}$ in 2010 to $6.87 \times 10^{4} \mathrm{t}$ in 2015 , a decrease of $25.9 \%$ (Table 4) [9].

\section{The development direction of green manufacturing in the chemical industry}

Through the analysis and discussion of joint technology and a model for a green manufacturing industry in China's chemical industry, the development direction of green manufacturing in China's chemical industry is proposed, including six aspects: green raw material, efficient process, green product, industrial recycling, production intelligence, and enterprise intensification.

\subsection{Green raw material}

The connotation of green raw material includes two aspects. First, in the design process of green chemical products, green and clean raw materials, including green ore, green solvents,

Table 1. Production of major chemical products in the chemical industry.

\begin{tabular}{lccc}
\hline Product & Production in $2010\left(\times 10^{4} \mathrm{t}\right)$ & Production in $2015\left(\times 10^{4} \mathrm{t}\right)$ & Growth rate $(\%)$ \\
\hline Methanol & 1574 & 3930 & 149.7 \\
Ethylene & 1419 & 1714 & 20.8 \\
Soda & 2029 & 2592 & 27.7 \\
Caustic soda & 2087 & 3028 & 45.1 \\
Phosphate rock & 6600 & 14200 & 108.8 \\
N, P, K and K fertilizer & 6337.86 & 7432 & 17.3 \\
\hline
\end{tabular}

Table 2. Main resource consumption intensity in the chemical industry.

\begin{tabular}{lccc}
\hline Product & Consumption intensity in 2011 (t/yuan) & Consumption intensity in 2015 (t/ yuan) & Growth rate $(\%)$ \\
\hline Phosphate rock & $1.28 \times 10^{-5}$ & $1.29 \times 10^{-5}$ & 0.8 \\
Pyrite & $1.6 \times 10^{-6}$ & $1.23 \times 10^{-6}$ & -23.13 \\
\hline
\end{tabular}

Table 3. Energy consumption of the main products in the chemical industry

\begin{tabular}{lccc}
\hline Product & Energy consumption in $2010(\mathrm{~kg}$ Standard coal $/ \mathrm{t})$ & Energy consumption in 2014 (kg Standard coal/t) & Decline $(\%)$ \\
\hline Ethylene & 880.7 & 816.3 & 7.31 \\
Calcium carbide & 1100 & 1010.2 & 8.2 \\
Synthetic material & 471.5 & 367.7 & 22.6 \\
\hline
\end{tabular}

Table 4. Pollutant emissions of the chemical industry.

\begin{tabular}{lccc}
\hline Pollutants & Emission of pollutants in 2010 (t) & Emission of pollutants in 2015 (t) & Decline $(\%)$ \\
\hline Solid waste & $1.396 \times 10^{5}$ & $6.07 \times 10^{4}$ & 56.5 \\
Waste water & $5.20 \times 10^{9}$ & $3.46 \times 10^{9}$ & 33.5 \\
COD & $3.28 \times 10^{5}$ & $2.43 \times 10^{5}$ & 17.7 \\
Nitrides & $9.27 \times 10^{4}$ & $6.87 \times 10^{4}$ & 25.9 \\
\hline
\end{tabular}


and green catalysts, are used to replace toxic, harmful, and stimulating raw materials. For example, recycled and reusable solid catalytic additions, enzyme catalysts, and bionic catalysts could be researched and developed [10]. Second, raw materials are pretreated by the process of homogenization. In the design of the whole process of the assembly of chemical products, the function, quality, development cycle, and cost of the product are fully considered. At the same time, the parameters of raw materials are optimized, so that raw materials can finish rough pretreatment, screening, and preprocessing before entering the transformation process, thereby reducing the fluctuation amplitude of the chemical components of materials. Thus, the overall stability of the chemical process is improved, the energy consumption of the subsequent production process is reduced, and the quality of the terminal products is guaranteed.

\subsection{Efficient process}

The high efficiency of the chemical process can not only improve the efficiency of production, but also overcome the problem of "high energy consumption, high pollution, and high material consumption." For example, supergravity desulfurization technology is used to treat the tail gas of a pyrite acid plant to improve desulfurization efficiency. Micro-chemical technology is used to improve the selectivity of cyclohexanone oxime catalyzed by sulfuric acid to produce caprolactam. Plasma technology is used in the acetylene process in coal. In addition to the above examples, magnetically stabilized bed technology, ionic liquid technology, supercritical fluid technology, and microwave radiation technology offer further application value in improving process efficiency. In the future, related technologies should be further studied and applied to achieve high efficiency in the chemical process.

\subsection{Green product}

Green chemical products should have two characteristics: (1) the products themselves do not cause health problems or environmental pollution; (2) the products can be recycled or degraded to harmless substances after use. In the design process of green chemical products, it is a requirement that the environmental impact is equal to the function of the product. The catalysts, extractants, plasticizers, etc. involved in the production process of the product should also be harmless, recyclable, and degradable. In addition, through the combination of chemical production and information technology, the function of a customized service is provided to improve the efficiency of product utilization and reduce the waste gas rate of products. For example, customized fertilizers are produced according to soil data, thus to provide targeted agrochemical services; and customized cosmetics are produced according to customers' requirements.

\subsection{Industrial recycling}

The sustainable development of China's economy and society increasingly faces serious constraints of a bottleneck of resources and the capacity of the environment. Domestic resources are hard to sustain as part of the sustained growth of the economy, and the environment is more difficult to support during the continuous expansion of the current chemical industry with the mode of high pollution, high consumption, and low efficiency in production. The development of China requires recycling of materials within the chemical industry, between the chemical industry and other industries, and between the chemical industry and society, to build key cross-industry projects, to build a cross-industry circular economy chain with the chemical industry as the core, to strengthen the comprehensive utilization of by-products and waste generated in the chemical production process, and to improve the efficiency of resource utilization [11].

\subsection{Production intelligence}

The information technology revolution dominated by computers and the Internet has had a profound impact on the manufacturing industry. Information technology in the petroleum and chemical industries is facing a new round of development opportunities. Under the influence of a new generation of information technology, such as the Internet of things, cloud computing, big data, and virtualization, China's chemical industry is stepping into an age of automation and networking. The deep integration of information and industrialization is key to the realization of intelligent manufacturing. The oil and chemical industries are typical process industries with complex processes and multiple control points. In particular, large refining and chemical projects are huge system projects. Intelligent chemical manufacturing can achieve a high amount of integration of horizontal, vertical, and end-to-end businesses, and enhance the level of plant operation management in a more precise and dynamic way, promoting the formation of new manufacturing and business mode innovations.

\subsection{Enterprise intensification}

There are many problems in China's chemical industry, such as the number of enterprises, the small scale, the dispersion of technology, the low strength of the technology integration, and so on, which means that the overall competitiveness of the industry is not strong. The construction of an eco-industrial park is an effective model for the development of a new type of industrialization, which is of great significance in solving structural and regional pollution, adjusting the industrial structure and layout, achieving energy savings and emission reductions, and building a resource-saving and environmentally-friendly society. In order to improve the overall competitiveness of the chemical industry 
in China and strengthen the links between the chemical industry and various other industries, the industrial layout of the chemical industry should be further improved, thus to realize intensified development of industries.

\section{Linking technology of the green manufacturing industry in the chemical industry}

With the rapid growth of the chemical industry, the large consumption of resources and energy constitutes the tightest bottleneck constraining the sustainable development of the economy and society. Therefore, recycling and reuse between the chemical industry, the chemical industry and other industries, and the chemical industry and society is an important way to achieve green manufacturing and sustainable development.

\subsection{The construction of the whole lifecycle industrial chain within the industry}

The chemical industry is an industry associated with high energy consumption and heavy amounts of pollution. In order to realize green manufacturing in the chemical industry, the concept of green development should run through the whole production process lifecycle of "resource-process-product-use."

\subsubsection{Resource.}

The efficiency of resource utilization should be improved. For example, in the phosphorous chemical industry, the utilization of medium and low grade phosphate ore, the technology of mineral enrichment and utilization, and the development of insoluble potassium resources should be strengthened, so as to enhance the utilization of medium and low grade minerals. In the petrochemical industry, carbon dioxide flooding technology should be used, which utilizes carbon dioxide to improve oil and gas recovery.

\subsubsection{Process}

New technology for energy saving and consumption reduction should be adopted to strengthen the water cycle of the chemical process and reduce the consumption of water, electricity, and energy. For example, in the calcium carbide industry, the use of the oxygen heat method in the process of synthesizing calcium carbide can reduce the consumption of electricity. In the coking industry, new dry quenching technology can efficiently recycle the waste from heat resources in the process of coking [12].

Comprehensive utilization of wastes in the chemical industry should be conducted. For example, waste gas recycling, such as utilization of flare gas for power generation, utilization of yellow phosphor furnace tail gas for recycling and producing monocarboatomic chemicals such as $\mathrm{CO}$ and $\mathrm{CH}_{4}$, and compre- hensive utilization of tail gas from the calcium carbide furnace, should be continued; wastewater recycling, such as extraction of industrial by-product salts from organic wastewater with high concentration of salt, recycling of ammonium sulfate and ammonium chloride from acid-containing wastewater, should also be continued; the centralized treatment and recycling of wastewater should be carried out to improve the utilization rate of water circulation, such as phenol wastewater treatment and reuse, high concentration salt water treatment and reuse.

\subsubsection{Product}

The performance of the product should be improved and upgrading of the product should be promoted, for example, in the chemical fertilizer industry, strengthening the development and upgrading of slow-release fertilizer, controlled-release fertilizer, bulk blending fertilizer (BB fertilizer), compound fertilizer, and flower-specific fertilizer for lawn products. In the carbide industry, calcium carbide furnace gas is purified by wet gas washing technology and dry cleaning technology to improve the utilization rate of calcium carbide furnace gas.

\subsubsection{Use}

According to the needs of the use, corresponding supporting services should be carried out. For example, in the chemical fertilizer industry, research into soil formula fertilization technology, precision fertilization technology, variable fertilization technology, and so on, should be conducted.

\subsection{Cooperative development of green manufacturing between industries}

The industrial structure should be adjusted and stronger cooperative and industrial links should be developed. Through the mutual utilization of waste, by-products, and products in the chemical industry and other industries, the organic connection between enterprises and industries, and the closed circulation of materials are realized, while the technical content and added value in the process of chemical production are improved [13].

(1) Waste and by-products in the chemical industry are used as raw materials for other industries. For example, in the phosphorous chemical industry, phosphogypsum produced during the production of phosphate fertilizer can be used for the production of a cement sustained-release agent, a light heat insulation material, and more, thereby building an industry chain of phosphorite-phosphate fertilizer-phosphogypsum-building materials. In the petrochemical industry, products of refined petroleum products and the recycling of waste mineral oil can be used as building materials. In addition to the above cross-industry collaborative connection, it is also possible to build many cross-industry collaborative industrial chains such as synthesis ammonia-gas-making furnace slag-building materials, coking- 
waste residue-cement, and refining-waste gas-heat and power supply.

(2) Waste and by-products from other industries are used as raw materials for production in the chemical industry. For example, in the iron and steel industry, blast furnace slag produced in the blast furnace process is used in the production of silicon, glass, and ceramics. In the mining industry, such as coal mining, coal produced in the process of coal gangue can be used for the production of crystalline aluminum chloride, ammonium sulfate, water glass, and other chemical products. The nonferrous metals industry contains large amounts of copper and nickel, chromium, iron, zinc, and other precious metals from electroplating sludge, which can be recycled for production of catalysts and tanning agents..

(3) Products from the chemical industry are used in other industries. For example, the industrial chain of polyvinyl chloride is extended to produce agricultural drip irrigation equipment and promote the development of modern and efficient agriculture, thus to form an industrial chain of calcium carbidePVC-water-saving equipment-high-efficiency agriculture-food processing-agriculture industrialization. Intelligent chemical fertilizers are produced to meet the needs of modern agriculture for fine, efficient, nitrogen, phosphorus, potassium, and a variety of balanced and different forms of chemical fertilizer. Chemical products, such as chemical fiber monomers, high quality chemical raw materials, dyes, and printing and dyeing auxiliaries are produced for the textile industry. For the photovoltaic industry, durability and flexibility are achieved when flexible thin film materials are used to achieve the size of thin film battery manufacturing.

(4) Products from other industries are used in the chemical industry. For example, the sulfur, phosphate beneficiation technology is used in the mining industry as a way of improving the chemical production process of resource utilization. In the process of chemical production, a large number of energy-saving motors and efficient energy-saving compressors have been introduced to reduce energy loss. Advanced control technology is used to reduce the fluctuation of process variables and improve the operation of devices and the stability of the product. Equipment and residual heat utilization technology have been introduced to save energy, reduce emissions, and lower production costs.

\subsection{Ecological link between industry and society}

In the development process of the chemical industry, industrial development should be organically combined with urban construction and ecological construction. Urban waste recycling and utilization projects should be carried out to utilize the resources of urban waste and improve the efficiency of the allocation of resources, along with the overall competitiveness of the city. For example, food waste and grassland waste are classified by microbiological technology to make compost. The food waste is sterilized and fermented at high temperature to produce feed additives or organic fertilizers. Straw is used to produce organic fertilizer, feed, and biofuel. Organic waste is used in urban life for the production of biogas. Waste batteries are recycled.

In addition, waste from the chemical industry, along with urban waste, should be treated in a unified way, and the layout of environmental protection facilities should be perfect for this task, while production and life service should be matched. For example, there should be a reliance on sewage and garbage disposal facilities in chemical production to solve the problems of domestic sewage and domestic waste treatment. We should reduce the construction of small "three wastes" treatment facilities, and rely on, utilize, and build large-scale "three wastes" processing systems as much as possible to improve the efficiency of waste disposal and promote the green development of the city.

\section{Typical cases of green manufacturing industry links in the chemical industry}

This section uses three cases to describe the specific application of the three types of industrial link patterns.

\subsection{Green manufacturing within industry}

Some phosphorus chemical industry group is a large enterprise in phosphate mining, phosphorus fertilizers, chemical processing of phosphorus sulfur coal, chemical production of fluoride and iodine, scientific researches, domestic and international trades, industry technology and operating services, and international project general contracting. According to the $3 \mathrm{R}$ principle of reduction, reuse and resource utilization, this group adopts advanced and practical technology to improve traditional industries, and constantly adjusts the industry and product structures, extending the industrial chain. At the same time, the efficiency of the economy is improved, the productivity of resources is increased, and the emission of pollutants is reduced.

The industrial chain of the group includes many sections (Fig. 2), such as phosphorite mining and processing, production and purification of wet process phosphoric acid, phosphate and fine chemical processing, phosphate fertilizer processing, and comprehensive utilization of resources. In the process of industrial chain transformation, a variety of new technologies have been adopted, such as sulphuric acid co-production of iron concentrate with low grade pyrite, medium and low grade phosphate ore dressing technology, high efficiency extraction and purification technology of wet process phosphoric acid, the cleaning technique of purification of diammonium phosphate (DAP) and co-production of monoammonium phosphate (MAP), new technology of crystal MAP, high-grade hydrated silica and ammonium fluoride technology, phosphate ore associated iodine 


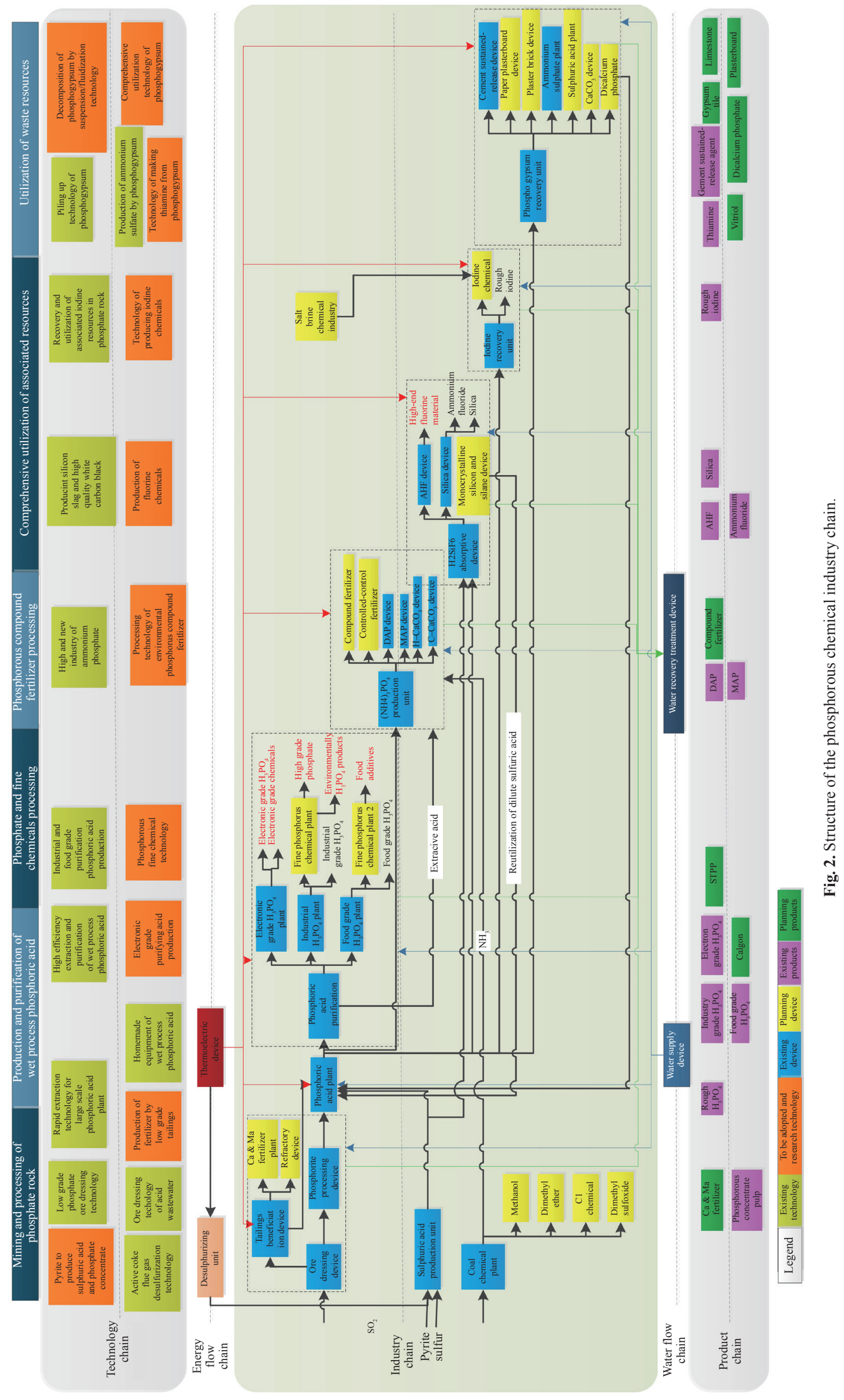


recycling technology, acid wastewater beneficiation technology, and comprehensive utilization of phosphogypsum.

Through the implementation of various technologies and projects, the industrial chain of the phosphorus chemical industry group has been extended effectively, and the structure of single phosphorus resource products has been developed to the structure of phosphorus, fluorine, iodine, and other multi-resource products. The utilization rate of resources has been greatly improved. The output value of phosphate rock, the recovery rate of phosphorus resources, the recovery rate of fluorine resources, and the recovery rate of iodine resources have been greatly improved. Comprehensive energy consumption for the production value per ten thousand yuan, and comprehensive energy consumption for products are lowered, and are in an international leading level within the industry. The industrial "three wastes" have been effectively harnessed, and the "zero discharge" of waste water has come to the forefront of the phosphate fertilizer industry.

\subsection{Cooperative development between industries}

The leading products of some new chemical fertilizer enterprise are compound fertilizers, slow controlled release fertilizers, water soluble fertilizers, and other new fertilizers. It has the largest production base of slow controlled release fertilizer in the world. Through popularizing the technology of seed and fertilizer co-sowing, formula fertilization by soil testing, and scientific fertilization, this group has formed a typical inter-industry link mode, that is, the link mode of the chemical fertilizer industry and new agriculture. In addition to new fertilizer, precision fertilization, and drip irrigation facilities, the group can also provide intelligent green pesticide products and feed for breeding, and provide techniques and services for recycling of forestry and agricultural residues such as straws, waste agricultural films, waste drip irrigation facilities, feces of livestock and poultry, and wastes produced in agro-product processing. The green development of the chemical fertilizer industry is combined with the demonstration of the modern agricultural recycling economy.

A PVC enterprise extends its industry and products to the upper and lower reaches, connects the chemical industry closely with other industries, and constructs two perfect industrial chains of circular economy [14]: an industrial chain of mineral resource exploitation-electric power-calcium carbide-PVC-water-saving equipment-high-efficiency agriculture-food processing-agricultural industrialization; and an industrial chain of recycling and reuse of industrial residues-cement building materials and waste drip irrigation belts. The two industry chains combine the PVC industry with modern agriculture. Targeting the current situation of regional agricultural development and the bottleneck of water shortage, the conversion efficiency of all kinds of resources in the chemical industry is greatly improved.

\subsection{Ecological links between industry and society}

Some fuel ethanol group is the most representative ethanol production enterprise with the longest history in China. In the fuel ethanol industry, this group has established an ecological connection through logistics (raw material wheat, flour, starch slurry, distiller's grains, etc.), forming a typical link mode between industry and society, that is, the linking mode between the biochemical industry and society. Starting from the wheat field, many eco-industrial chains, such as wheat field $\rightarrow$ bran production enterprise $\rightarrow$ vital gluten company $\rightarrow$ fuel ethanol company $\rightarrow$ DDG company $\rightarrow$ methane company $\rightarrow$ organic fertilizer company $\rightarrow$ wastewater treating company, and fuel ethanol company $\rightarrow \mathrm{CO}_{2} \rightarrow$ degradable plastics company, are formed, and each chain ends with an environment comprehensive treating system. Each of the eco-industrial chains is interlaced and coupled with each other through the flow and sharing of material, energy, and information to form a fuel ethanol eco-industrial system.

\section{Conclusions}

Based on the current situation of green manufacturing in China's chemical industry, the development direction of green manufacturing in China's chemical industry is put forward. On this basis, the model and technology for building the industrial chain of chemical green manufacturing are discussed, and the industrial link mode is expounded through typical cases. In addition to the construction of an industrial chain in the chemical industry, the chemical industry should also be organically linked and coordinated with other industries and societies through products, by-products, wastes, etc. Referring to the development model of typical enterprises, green development is realized in many aspects, such as raw material, process, product, industry, production, enterprise, and so on.

\section{References}

[1] Wang F, Sun D Q. Strategy of green manufacturing in chemical machinery industry [J]. China Petroleum and Chemical Standard and Quality, 2013 (22): 39. Chinese.

[2] Feng F. Promote industrial greening, promote industrial restructuring and development $[\mathrm{J}]$. Chinese Informatization, 2016 (2): 7-9. Chinese.

[3] Chen X L. Present situation and development of green chemistry and chemical engineering [J]. Heilongjiang Science, 2014, 5(3): 282. Chinese.

[4] Hu S Y, Chen D J, Jin Y, et al. Research on green development strategy of chemical industry: Based on the analysis of chemical fertilizer and coal chemical industry [J]. Journal of Chemical Industry and Engineering (China), 2014 (7): 2704-2709. Chinese.

[5] Liu L. Modernization of China's chemical industry facing green manufacturing [J]. Science and Modernization, 2015 (4): 9. Chinese. 
[6] Jing B X, Ju F. Application of green chemical technology in chemical engineering process [J]. Tianjin Chemical Industry, 2015 (3): 10-11. Chinese.

[7] Yao J H, Xu W L, Jiang S Y, et al. Application of intelligent technology in green chemical industry [J]. Shanghai Chemical Industry, 2016 (10): 19-26. Chinese.

[8] National Bureau of Statistics. 2016 China industry statistical yearbook [M]. Beijing: China Statistics Press, 2016. Chinese.

[9] National Bureau of Statistics. 2011 China statistical yearbook on environment [M]. Beijing: China Statistics Press, 2011. Chinese.

[10] Govindan K. Green sourcing: Taking steps to achieve sustainability management and conservation of resources $[\mathrm{J}]$. Resources
Conservation \& Recycling, 2015 (104): 329-333.

[11] Hao Q, Tian J P, Li X, et al. Using a hybrid of green chemistry and industrial ecology to make chemical production greener [J]. Resources Conservation and Recycling, 2017 (122): 106-113.

[12] Shangguan F Q. Scientific strategy of coking industry green development [J]. Iron \& Steel, 2015, 50(12): 11-18. Chinese.

[13] Tian J, Guo Q, Chen Y, et al. Study on industrial metabolism of carbon in a Chinese fine chemical industrial park [J]. Environmental Science \& Technology, 2015, 47(2): 1048.

[14] Cao T Z. Green manufacturing and green rebuilding in chemical industry [J]. General Machinery, 2013 (9): 26-28. Chinese. 